\title{
NORMATIVIDAD Y DISCONFORT DE GÉNERO: ESTRATEGIAS Y RESISTENCIAS EN LA FAMILIA CONTEMPORÁNEA
}

\author{
NORMATIVITY AND GENDER DISCOMFORT: STRATEGIES AND \\ RESISTANCES EN CONTEMPORARY FAMILY
}

\author{
Alejandra Martínez \\ CEA-CONICET \\ Universidad Nacional de Córdoba. Argentina/Argentina \\ martinezalej@hotmail.com
}

Recibido/Received: 09/11/2010

Aceptado/Accepted: 01/08/2011

\section{RESUMEN}

El presente artículo es producto de un trabajo de investigación realizado entre 2006 y 2009, que tuvo como uno de sus objetivos principales analizar las representaciones de las normas de género en varones y mujeres provenientes de diferentes condiciones objetivas de existencia. En dicho estudio se observó que, cuando las representaciones de género se alejan o contraponen con aquellas que son producto de las condiciones objetivas en las que se gestaron los habitus, las mujeres y los varones tienden a manifestar discursivamente una "sensación de incomodidad" respecto de las regulaciones de género que los constriñen. En el artículo se avanza sobre la explicación y sistematización de dicha sensación, denominada teóricamente como "disconfort de género".

PALABRAS CLAVE

Disconfort de género, normas de género, representaciones, investigación cualitativa.

\section{SUMARIO}

1. Introducción. 2. Diseño metodológico. 3. El disconfort de género: hacia una definición del concepto.

4. Reflexiones finales. Bibliografía

\begin{abstract}
This article is product of a research work developed between 2006 and 2009. One of its main objectives was to analyze gender norms' representations of men and women that came from different objective conditions of existence. In the study we observed that when gender representations contrast those that are product of the objective conditions in which habitus were developed, women and men tend to manifest in their discourses a sort of "discomfort sensation" related to those gender regulations that limit their practices. In the article we advance on the explanation and systematization of such sensation called, theoretically, "gender discomfort".
\end{abstract}

\section{KEYWORDS}

Gender discomfort, gender norms, representations, qualitative research.

\section{CONTENTS}

1. Introduction. 2. Methodology. 3. Gender discomfort: towards a definition of the notion. 4. Final thoughts. References. 


\section{INTRODUCCIÓN}

Este artículo es producto de un trabajo de investigación realizado entre 2006 y 2009, que tuvo como uno de sus objetivos principales analizar las representaciones de las normas de género en varones y mujeres provenientes de condiciones objetivas diversas.

Las normas de género son regulaciones consideradas legítimas que se orientan a definir prácticas, expectativas y maneras de comportarse, para varones y mujeres. Estas regulaciones se fundamentan en ejes de sentido que oponen lo femenino y lo masculino.

Relacionados a lo femenino surgen sentidos tales como el rol de reproducción, la pasividad, lo privado, lo emotivo, entre otros. Y en relación a lo masculino, encontramos sentidos vinculados al rol de producción, la actividad, lo público, lo racional, etc. (Fraser, 1997, 2000; Mayobre, 2004; Bourdieu, 2000).

Ahora bien, tales sentidos asociados con lo femenino y lo masculino parecen presentar ciertas variaciones de acuerdo a las condiciones objetivas de existencia de los agentes sociales. Dicho esto, surge la necesidad de precisar la noción de "condiciones objetivas de existencia".

Desde nuestra perspectiva, este concepto excede largamente la idea de clase, ya que incluye las condiciones estructurales que tienden a condicionar las prácticas sociales y que son ajenas a la voluntad de los agentes. Así, además de la capacidad de relación que otorga la posesión o privación de capitales económicos y culturales (Bourdieu, 1999, 1988), son condiciones objetivas el género, la etnia y la estructura familiar, entre otros (status adscritos). Estas condiciones señalan espacios de posibilidad y orientan prácticas sociales, de este modo, no resulta lo mismo ser mujer, ser mujer y de clase baja, o ser mujer, inmigrante, de clase baja y depender del ingreso del marido.

Las condiciones objetivas otorgan a los agentes sociales una mayor o menor posibilidad para poder pensarse en espacios de posibles diversos, y le otorgan capacidades diferenciadas de relación (Mozejko y Costa, 2002).

Las condiciones objetivas de existencia que identificamos en este estudio como más influyentes en la variación de las representaciones sobre el género son cuatro:

a- El género mismo

b- La posesión de capitales económicos y culturales

Para evitar confusiones entre el concepto de "clase" y el de "condiciones objetivas de existencia", hemos construido tres categorías de acuerdo con el volumen y estructura de recursos económicos y culturales que posee un sujeto o conjunto de agentes sociales. A estos los identificamos como grupos "Alto", "Medio" y "Bajo".

c- La estructura de ingresos en el interior de la pareja

El modo en que se divide el "poder" en el seno de la unidad familiar a partir del aporte diferencial de capitales de cada miembro de la pareja es un aspecto clave a considerar dentro del análisis de discursos producidos en torno al género. Cuando ambos miembros de la pareja trabajan en espacios laborales similares u "homogámicos", los dos aportan los ingresos centrales en el hogar. Esto reduce la dependencia de las mujeres y les brinda una mayor capacidad de tomar decisiones en la familia. Si la mujer tiene una posición relativa superior a la del varón ("hipogámica") su capacidad de acción y decisión también se incrementa (Carbonero Gamundí, 2007). La situación tiende a ser diferente cuando el marido tiene una posición superior en la pareja ("hipergamia"). En tal caso, el aporte que proviene 
del trabajo de la mujer es conceptualizado como complementario y sujeto a su rol de reproducción.

En nuestro trabajo, el aporte diferencial de recursos no se refiere sólo al capital económico, sino también al nivel de educación alcanzado (capital cultural institucionalizado), o las características que tiene el empleo de los miembros de la pareja.

d- El marco familiar en el que se gestaron los habitus de los agentes sociales (familias de origen más bien tradicionales o más bien innovadoras)

$\mathrm{Al}$ analizar la relación entre representaciones y condiciones objetivas de existencia, observamos que cuando dichas representaciones se alejan o contraponen con aquellas que son producto de las condiciones objetivas en las que se gestaron los habitus, mujeres y varones tienden a manifestar una "sensación de incomodidad" respecto de las regulaciones de género. Hemos denominado esta incomodidad "disconfort de género". El objeto de este escrito es explicar en qué consiste dicha noción.

\section{DISEÑO METODOLÓGICO}

\subsection{Método y técnica aplicados}

En el presente trabajo de investigación se implementó la metodología cualitativa, inserta en el paradigma constructivista (Valles, 2003; Olabuénaga, 2003). La metodología cualitativa resultó fundamental para acceder a las representaciones de las normas de género de los agentes sociales e identificar las condiciones objetivas a partir de las cuales se gestaron los habitus que produjeron tales representaciones.

La técnica de recolección de datos utilizada fue la entrevista en profundidad, cuya ventaja radica en la flexibilidad que ofrece su aplicación y en la posibilidad de permitir la emergencia de representaciones no previstas en la indagación, que pueden resultar de interés para los objetivos del trabajo. La entrevista en profundidad presenta, además, la característica central de ofrecer un entorno de diálogo y de indagación permanente, lo que permitió orientar y reorientar los contenidos de las conversaciones con los entrevistados en función de la dinámica que las mismas fueron asumiendo (Merlino, 2009).

El instrumento utilizado para movilizar el diálogo en las entrevistas fue la "guía de pautas". Los temas centrales contenidos en la guía se mencionan a continuación:

- Descripción de la vida personal y familiar (costumbres, actividades)

- Representaciones en torno al trabajo doméstico y asalariado

- Caracterización de hijos e hijas, expectativas en torno al futuro de los mismos

- Representaciones respecto de "ser varón/mujer"

Dado el planteamiento de los temas mencionados como punto de partida de las conversaciones en las entrevistas, se organizó una guía que abarcase dichos temas y los presentara de modo organizado y en una secuencia inicial que podía variar según fuera dándose el diálogo.

\subsection{Población de estudio y procedimiento muestral}

Los actores de interés para el trabajo fueron varones y mujeres de entre 20 y 45 años, residentes en la ciudad de Córdoba, Argentina, padres y madres de niños y niñas no mayores a 12 años. Para la selección de los agentes sociales a entrevistar se utilizó un muestreo por 
propósitos y por saturación de categorías, en el que se tuvo especialmente en cuenta la heterogeneidad y accesibilidad.

La muestra por propósitos posibilitó establecer comparaciones e identificar las semejanzas y diferencias entre individuos provenientes de condiciones objetivas diversas (Maxwell, 1996). La utilización de este tipo de muestra permitió seleccionar aquellos casos que consideramos típicos (o pertinentes), por alguna característica particular, y que resultaban de interés en función de los objetivos planteados.

La elección de las edades de los entrevistados -entre 20 y 45 años- se basó en la opción de trabajar con personas que hubieran nacido no antes de la década de 1960. En las décadas del '60 y '70 cobró relevancia, en todo el mundo occidental, la Segunda Ola feminista que trajo profundos cambios a la realidad de las mujeres y la vida familiar y social en general. Por ello fue de interés analizar las representaciones de agentes sociales nacidos y criados en épocas posteriores a que se produjera la Segunda Ola por estar expuestos, desde su infancia, a discursos y prácticas sociales emergentes en torno a las definiciones del género.

La decisión de que los entrevistados tuvieran hijos e hijas no mayores a 12 años, respondió a la necesidad de evitar que las expresiones de los agentes sociales se centraran en las problemáticas de la sexualidad durante la adolescencia, y que pudieran expresar de un modo más "proyectivo" sus expectativas en relación con las definiciones de género de sus hijos e hijas.

Siguiendo la propuesta del muestreo teórico (Strauss; Corbin, 2002; Vieytes, 2004), a lo largo del trabajo se produjeron modificaciones en la selección de los entrevistados así como también en la guía de pautas en función de que, en la medida en que surgían temas que resultaban de interés, se agregaban ítems en la guía o casos a indagar.

La muestra alcanzó los 32 casos, 16 representados por mujeres y 16 por varones. Estos fueron relevados en dos etapas; una al comienzo del proyecto (16 casos) y otra cuando ya se habían analizados los primeros datos de campo (los 16 casos restantes). Los criterios para la selección de los agentes sociales entrevistados se basaron en la accesibilidad y la representatividad tipológica.

En este trabajo participaron entrevistadores varones con el objeto de reducir al máximo la percepción de evaluación. Sin embargo, entendemos que la asimetría y la sensación de ser observado del sujeto entrevistado, durante la situación de entrevista, es casi inevitable (o bien, inevitable). En este y en otros casos, nos ha sido útil para analizar señales que dan cuenta de situaciones vergonzantes que para el entrevistado resulta difícil "confesar".

\section{EL DISCONFORT DE GÉNERO: HACIA UNA DEFINICIÓN DEL CONCEPTO}

A partir de los años 60, el progresivo avance de las mujeres en el campo productivo les trajo como consecuencia la posibilidad de adoptar roles asociados con el género que no estaban vinculados a sus aprendizajes, en cuanto al lugar que les correspondía ocupar, ni a las aspiraciones que debían tener. Las mujeres se veían coaccionadas por regulaciones relativas a su sexo biológico incorporadas en sus habitus, que daban cuenta de su espacio de posibles; a la vez, veían cómo se abría ante ellas progresivamente un espacio de producción y actividad que les había sido presentado hasta entonces como ajeno.

Las condiciones objetivas relacionadas con la socialización del género se contraponían con los nuevos horizontes de posibilidades y expectativas. Era esperable que la inercia 
propia del habitus tendiera a perpetuar al menos los rasgos principales de aquellos esquemas incorporados, relacionados con lo que representaba socialmente "ser mujer".

Pero los progresos femeninos que se produjeron desde la Segunda Ola hasta el presente no lograron romper definitivamente con las clasificaciones tradicionales relativas a las normas asignadas a uno y otro género. Aunque se han producido avances muy significativos en relación a los espacios que las mujeres ocupan en la vida pública y en el espacio laboral, las representaciones que ubican en ejes opuestos y complementarios lo femenino y lo masculino, han permanecido relativamente inalteradas en el tiempo (Bourdieu, 2000).

Según lo que pudimos observar entre las mujeres entrevistadas en la investigación y, sobre todo, entre aquellas que se muestran más comprometidas con su nuevo rol productivo/ público, surge una suerte de "incomodidad" que parece estar asociada con el extrañamiento que generaría alejarse del rol femenino tradicional al adentrarse en un rol (masculino) aprendido como en el marco de lo "impensable". Esta incomodidad también se observa entre aquellos varones cuyas parejas se encuentran fuertemente comprometidas con el rol productivo y, sobre todo, si ellas perciben un ingreso mayor al de ellos. Esta situación pone en cuestión el espacio masculino tradicional: el de principal proveedor y protector de la familia.

Llamamos a esta incomodidad, tanto masculina como femenina, "disconfort de género".

El disconfort de género surge en los agentes sociales cuando, por las actividades que realizan o el tipo de pareja que han conformado (hipergámica, hipogámica, homogámica) se alejan de los polos normativos de género que han aprendido como propios, o perciben que la pareja avanza sobre el espacio social que les corresponde poniendo en riesgo su lugar. A su vez, este alejamiento del espacio propio tiene que ver, en la mayoría de los casos, con un acercamiento a los lugares aprehendidos como ajenos. Los agentes sociales de ambos sexos manifiestan el disconfort con expresiones como, por ejemplo, "estar en falta", en el caso de las mujeres, y de incertidumbre (respecto de su rol), en el caso de los varones.

El disconfort se impone a los agentes sociales en dos dimensiones: una "interna", que tiene que ver con los habitus, la crianza y la reproducción de normas tradicionales incorporadas como válidas. Y la otra es una dimensión "externa" que se materializa en las voces reales y concretas de familiares y congéneres que señalan el incumplimiento de las regulaciones de género naturalizadas. Estas voces reclaman, a través de la burla o la recriminación, el cumplimiento de normas de género que son evaluadas como adecuadas. En el caso de las mujeres, uno de los reclamos más recurrentes es, por ejemplo, dedicar más atención a los hijos. En el caso de los varones, no cumplir con el rol de proveedor único o principal del hogar puede representar el señalamiento más importante. Pero también surge otro tipo de reclamo que insta al abandono de tareas poco masculinas -y por lo tanto inadecuadas-, tales como realizar quehaceres domésticos para ayudar a las mujeres.

Véase, en la Figura 1, un esquema que da cuenta de las dimensiones en las que se presenta el disconfort.

En el caso de las mujeres, el disconfort de género se produce cuando ellas perciben como enfrentadas dos demandas a las que asignan valencia positiva: a) la necesidad de hacer cosas "fuera" de la casa que le otorgan satisfacciones personales (económicas, vocacionales, de evasión, entre otras) y b) la responsabilidad sobre el espacio reproductivo, que han aprehendido como propio y que entienden que les corresponde por excelencia.

Este último puede por momentos pesarles, abrumarlas, pero a la vez adquiere un valor particular al identificarlas como "verdaderas mujeres", valoración incorporada que redunda 
en requerimientos sociales que las señalan "en falta" cuando incumplen su rol "natural" femenino/materno.

Figura 1. Dimensiones del disconfort de género

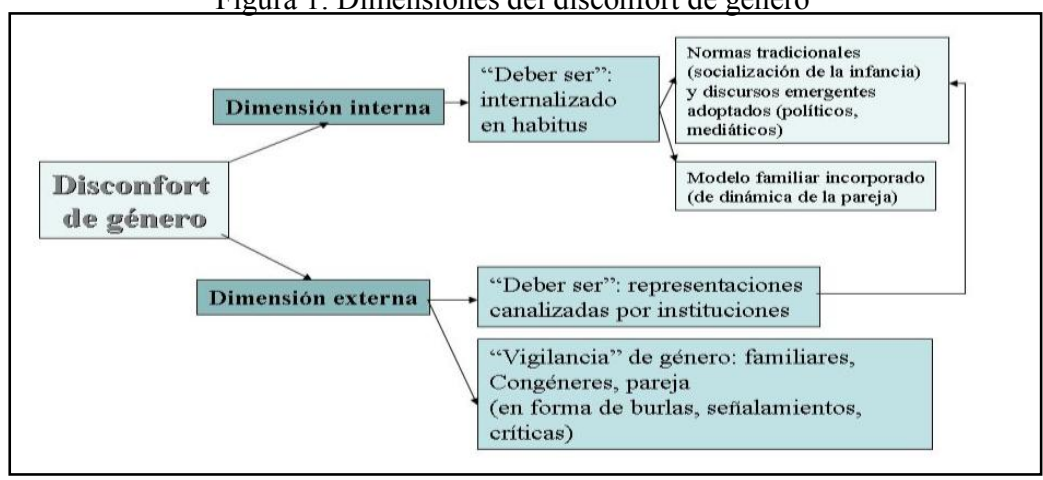

La incomodidad en las mujeres se muestra cuando dicen sentir que deben "abandonar" a los hijos para dedicarse al trabajo, dejar de atender al marido y el "interior" de la casa para "salir" a trabajar (como los hombres). En estos casos, las mujeres manifiestan cargar con la "culpa" de alejarse del polo de la reproducción, por acercarse al de la producción.

En el caso de los varones sucede algo similar. Históricamente se ha esperado de ellos que se hagan cargo de sus familias, sacrificándose por la mujer y los hijos, demostrando a la sociedad su valía masculina a partir del éxito profesional o económico como metas fundamentales. Ganar la verdadera masculinidad ha representado para los varones ganar dinero, demostrar su capacidad de ejercer la violencia, defender y defenderse, y hacerse respetar o tener poder sobre las cosas y personas de su entorno. Todas estas características, mayormente vinculadas a los sentidos de productividad y actividad, no parecen haber resultado cargas livianas. Sin embargo, el hecho de que la esposa comparta con ellos la responsabilidad de sostener el hogar se habría convertido en algo negativo al amenazar el espacio de identificación masculino por excelencia: la producción. El disconfort de género en este caso se presenta bajo la forma de insatisfacción o frustración por no ser capaces (en la fantasía) de garantizar la supervivencia de la familia, sin ayuda de la mujer.

\subsection{Los grados en los que se manifiesta el disconfort de género}

Parece haber gradientes en relación al disconfort de género, es decir, grados en que la incomodidad se manifiesta. Ello parece depender fundamentalmente de dos condiciones:

a) El modo en que se distribuyan los poderes al interior de la pareja (hipergamia/ hipogamia/ homogamia)

b) El grado en que las normas de género tradicionales (basadas en oposiciones de sentido) se encuentran cristalizadas en los habitus de los agentes sociales.

Podemos identificar así tres diferentes grados de disconfort de género, tanto para varones como para mujeres.

En el caso femenino, un disconfort de género "alto" aparece cuando la mujer trabaja muchas horas al día, y por motivos que se encuentran más ligados a la elección personal que a la necesidad económica. Las condiciones objetivas a partir de las que se formó el habitus, que definen aspiraciones subjetivas como "ser una buena madre", se contraponen con las expectativas femeninas de disfrutar de la actividad laboral, o bien, de desarrollarse en un 
ámbito laboral. En este caso, el disconfort de género se presenta fuertemente con el formato de "estar en falta", y en dos dimensiones:

a) "Internamente": por desarrollar una actividad fuera del hogar se está " "abandonando los hijos por razones egoístas"

b) "Externamente": por los reclamos explícitos que provienen, en primer lugar, de la propia familia (el marido y los hijos) por no pasar más tiempo en la casa o realizar determinadas tareas de cuidado, y en segundo lugar, de congéneres y familiares (amigas, madre, suegra, entre otros).

Un aspecto adicional se suma cuando la mujer, además de trabajar muchas horas, gana más dinero que su pareja, "desplazando" al varón de su propio espacio de comodidad e identidad masculina, basada en ser el sostén principal.

Un disconfort de género de grado "medio" se da en el caso de aquellas mujeres que trabajan fuera de la casa, pero en jornadas muy reducidas. Además, cuando la actividad laboral se encuadra fundamentalmente en la satisfacción de necesidades de tipo económico, más que de gusto por la actividad que se realiza. Aún cuando estas mujeres han incorporado en sus habitus normas de género tradicionales, la justificación (interna y externa) de tener que trabajar para que el hogar sea sustentable, aminora el grado de disconfort que manifiestan sobre su doble rol. En el caso mencionado, las condiciones objetivas que tienden a ubicar el lugar natural de la mujer en el rol reproductivo, se contraponen en forma relativa con las expectativas subjetivas de ganar dinero para sostener el hogar. La limitación en la contradicción está dada por la "inevitabilidad" del trabajo femenino, para satisfacer las necesidades del hogar o para progresar económicamente. Esto disminuiría la noción de estar en falta por dejar a los hijos solos, y la idea de ser criticadas (por unos otros que vigilan) por no dedicarse tiempo completo a la casa.

El disconfort de género "bajo" se da cuando las condiciones objetivas en las que se gestaron los habitus (orientados a normas tradicionales de género) son coherentes con la situación presente que vive el agente social. En estos casos, la mujer no tiene la idea de estar en falta, y tampoco se percibe observada por un entorno crítico respecto del lugar que ocupa o las prácticas que realiza. Así es que, para el caso femenino, el disconfort parece ser menor entre las mujeres que no trabajan fuera de casa, o que trabajan pocas horas y sin salir de sus casas.

En la Figura 2 presentamos ejemplos en los que las mujeres manifiestan alguna de las dimensiones del disconfort de género (interna o externa) en diferentes grados.

En lo que respecta a los varones, el disconfort de género también tiene gradientes. El grado de disconfort es más elevado cuando los varones aportan al hogar menos que las esposas. Y aquí sucede algo opuesto al caso de las mujeres; para ellas, trabajar por la necesidad de sostener la casa parece representar un disconfort de género menor (ya que hay razones objetivas e ineludibles para hacerlo). Para los varones, que las mujeres tengan que trabajar para aportar al hogar representa aún más incomodidad. La situación en la que las mujeres adquieren importancia en el espacio de producción, se contrapone con representaciones tradicionales sobre el género que la mayoría de los varones ha incorporado a lo largo de su vida.

El nuevo lugar de la mujer es evaluado en términos de riesgo de ser reemplazado en el rol productivo. En el aspecto externo, de vigilancia social, el varón siente disconfort por ser evaluado (y hasta increpado) por un entorno que no aprueba su desplazamiento como principal sostén. 
Figura 2. Grados del disconfort de género en mujeres

\begin{tabular}{|c|}
\hline Grados del Disconfort de género en mujeres \\
\hline $\begin{array}{l}\text { Grado alto } \\
\text { "o sea, me duele un montón venirme tantas horas acá (trabajo) y dejarlo al bebé, me mata. Es } \\
\text { un bebé que todo el tiempo te dice 'gracias' con... (se le quiebra la voz, se hace un silencio) } \\
\text { aprendió hace poco y hacés algo y... 'gracias'... y capaz que porque no me ve en todo el dia } \\
\text { (llora)" (Ema, } 23 \text { años, trabaja tiempo completo, grupo Medio) }\end{array}$ \\
\hline $\begin{array}{l}\text { Grado medio } \\
\text { "hoy la situación económica hace que sea necesario que trabaje tanto el hombre como la } \\
\text { mujer por eso creo que es mucho más dificil el rol o el papel de la mujer en la familia (...) los } \\
\text { dos trabajamos, los dos criamos a nuestro bebé, si bien yo hago la mayor cantidad de cosas } \\
\text { en la casa Carlos cuando puede me ayuda" (Emilia, } 28 \text { años, trabaja medio tiempo, grupo } \\
\text { Medio) }\end{array}$ \\
\hline $\begin{array}{l}\text { Grado bajo } \\
\text { "(ser ama de casa) no es una obligación, lo hago con gusto o sea, por ahi si reniego 'tengo } \\
\text { que lavar toda esta ropa!' Pero todavia no lo vivo, como, ino por Dios! todavia lo hago con } \\
\text { ganas con gusto, pero... no lo veo como una obligación, si bien si, para tenerlas a las chicas } \\
\text { bien, la comida, las cosas a horario, pero no me parece algo nat... algo de la vida" (Maria, } 24 \\
\text { años, ama de casa, grupo Bajo) }\end{array}$ \\
\hline
\end{tabular}

También es alto el disconfort cuando los varones perciben que sus congéneres se burlan de ellos por realizar tareas domésticas para ayudar a la mujer. En esta situación, la incomodidad que los varones manifiestan se potencia al exponerse al juicio y repudio de aquellos "otros" que "vigilan" la legitimidad de la masculinidad, y ante quienes no se desea perderla.

Estas situaciones de disconfort generan discursos de resistencia, en defensa de los valores y los roles tradicionales (se trata de los casos en los que los varones afirman que desearían que las mujeres trabajaran menos, o bien, que no trabajaran en absoluto.).

En este caso, las condiciones objetivas en las que se gestaron los habitus contrastan fuertemente con la representación de la situación presente (y el lugar ocupado), por lo que el ajuste del habitus está lejos de producirse; por el contrario, la resistencia masculina tiende a cristalizarse aún más, enquistándose.

El disconfort de género masculino en grado "medio" se da en dos situaciones:

a) Cuando la mujer trabaja fuera de la casa media jornada, y más aún, si el dinero que gana no es significativo (en relación con el que aporta el varón). En esta situación, y a pesar de que aún nos referimos a varones que sostienen representaciones de normas de género tradicionales, el disconfort no es tan intenso como cuando la mujer aporta su ingreso para sostener el hogar. Al ganar significativamente menos dinero que el varón, el rol productivo de la mujer no atenta tan directamente contra el espacio masculino. Algo similar sucede si la mujer trabaja no por necesidad económica del hogar, sino por decisión personal. La idea de que el salario masculino es suficiente para mantener a la familia parece reducir el disconfort del varón.

b) Cuando el varón ha recibido un tipo de crianza mínimamente orientado a realizar algunas actividades domésticas (ordenar su habitación, prepararse la comida, etc.).

En los casos de disconfort de grado medio la salida de la mujer al mercado laboral (y el alejamiento -aunque sea por pocas horas- del hogar) produce quejas en los varones, pero no manifiestan un disconfort tan alto, pues no se disputa el rol productivo principal. Una de las justificaciones que surge más frecuentemente en los discursos que corresponden a estos casos, es la que refiere a la "justicia" en relación con los derechos de la mujer (de trabajar, 
desarrollarse, etc.). Aquí el aspecto externo, el de vigilancia social, no es tan intenso para el varón como en los casos de disconfort alto. Comenzaría a serlo en caso de que la mujer recibiera en su trabajo, por ejemplo, un ascenso, y la importancia de su puesto superara simbólicamente al del marido. Esto es observable en las parejas en las que la mujer ha alcanzado una mayor formación académica que el varón.

La segunda situación mencionada, la de los varones que han recibido una crianza con mayor apertura a las tareas de reproducción, es la menos frecuente. Estos varones gestaron sus habitus en marcos familiares en los que aprendieron a realizar algunas de las tareas del hogar y no viven con demasiada extrañeza la domesticidad. Aún sosteniendo representaciones tradicionales de género, y aunque la mujer trabaje fuera de la casa, parecen asumir con menor ajenidad su rol como "ayudantes" en las tareas de reproducción y, por ello, su disconfort de género no parece ser tan elevado como en los casos presentados anteriormente.

El disconfort de género se presenta como "bajo" (o nulo) cuando existe una coherencia casi absoluta entre las condiciones objetivas en las que se generaron los habitus masculinos, y la situación actual en la que cada miembro de la pareja se adecua a ese destino "natural": la mujer no trabaja y el varón asume el rol de proveedor y protector único de la familia. Esto representa, en términos de vigilancia del entorno, una mínima o nula sensación de reprobación social para el varón, ya que se encuentra ubicado en un espacio masculino por excelencia (y tal espacio no se ve amenazado).

Presentamos, en la Figura 3, ejemplos de varones que manifiestan disconfort de género:

Figura 3. Grados del disconfort de género en varones

\begin{tabular}{|c|}
\hline Grados del Disconfort de género en varones \\
\hline $\begin{array}{l}\text { Grado alto } \\
\text { "Al no tener trabajo, empieza a haber roce y... y que '¿por qué no vas a buscar trabajo?' (...) } \\
\text { es roce constante, no solamente con tu pareja, sino cuando vas a la casa de tu mamá, o a la } \\
\text { casa de tus suegros y sigue siendo roce" (Adrian, } 24 \text { años, grupo Bajo) }\end{array}$ \\
\hline $\begin{array}{l}\text { Grado medio } \\
\text { "(se refiere a lo que le gusta de su esposa) es lindo que una mujer quiera tener metas, y } \\
\text { defender esas metas, inocente, la misma palabra me lo dice, que me necesita a mi, a veces, } \\
\text { para llegar a esa meta, no depende de mi, pero me necesita, me necesita para explicarle" } \\
\text { (Carlos, } 32 \text { años, grupo Alto) }\end{array}$ \\
\hline $\begin{array}{l}\text { Grado bajo } \\
\text { "(mi señora) trabajaba en un cotillón, estuvo como } 4 \text { años... pero si no hubiera nacido el nene } \\
\text { ella estaria laburando, porque le gusta trabajar... y en la casa ella también está laburando, } \\
\text { porque está haciendo mis cosas, haciendo las cosas de la casa (...) y es importante porque } \\
\text { me tiene todas las cosas listas que yo quiero (..) le digo lavame las zapatillas', si o si, viernes } \\
\text { y sábado, 'lavame las zapatillas para el viernes y el sábado, las quiero tener limpias." (Diego, } \\
21 \text { años, grupo Medio) }\end{array}$ \\
\hline
\end{tabular}

\subsection{Reduciendo el disconfort: las prácticas discursivas}

Para reducir ante terceros el disconfort de género, varones y mujeres ponen en juego en la interacción con el/la entrevistador/a estrategias discursivas que apuntan a disminuir la incomodidad, al menos en la dimensión externa. Dado que el disconfort es más elevado cuando el rol de producción/sostén del hogar pasa a depositarse en la actividad femenina, es en esos casos en donde se observa más frecuentemente la búsqueda de disminución de la incomodidad. 
En cuanto a las estrategias discursivas específicas, observamos que las mujeres que tienen un ingreso mayor que el de los maridos tienden a señalar que no por ganar menos dinero el varón pierde su lugar como jefe del hogar. De esta manera, ellas buscan rescatar y reproducir discursivamente una estructura familiar típica, en la que el varón tiene la responsabilidad de ser el sostén principal de la economía familiar (dado que allí reside su valor). Por ejemplo:

"No, no me va eso de la mujer allá arriba y el hombre un mueble más de la casa, por más que lo sea ¿eh? (...) no me gusta, creo que si me tocase a mi, si fuera mi caso, que... no sé si lo es... porque si me pongo a sumar... no sé si lo es, pero no me gustaría eso... lo que yo quiero no es eso. Es el hombre, la mujer y los hijitos y somos familia" (Sol, 32 años, hipogamia, grupo Bajo)

En el caso de los varones encontramos que los que han constituido parejas hipogámicas tienden a "emparejar" discursivamente las aportaciones de uno y otro miembro de la pareja, y también a justificar con detalle el por qué de tal situación de distribución. Es observable que los agentes sociales que componen parejas hipogámicas (tanto varones como mujeres), intentan durante las entrevistas hacer pasar inadvertida esta condición objetiva que parece escapar a una "normalidad" aprendida y validada socialmente.

"En realidad andamos parejito ¿si? andamos más o menos los dos ganamos similar ¿si? Ella hoy en día está ganando un poco más pero bueno, porque le han dado unas materias a distancia ¿viste? Entonces hoy en día ella está ganando un poco más, pero no sé si llamarlo como el sostén... en realidad los gastos son... lo que son gastos comunes se reparten equitativamente" (José, 33 años, hipogamia, grupo Medio)

El énfasis discursivo puesto por el entrevistado en su intento de emparejar los ingresos de ambos miembros de la pareja, constituye una defensa de la integridad de su rol como jefe del hogar, aunque en la práctica no sea el principal sostén económico de la familia. Se percibe el disconfort de género ante el hecho de que su esposa aporte más dinero al hogar que él; más aún teniendo en cuenta que la persona que lo está entrevistando es otro varón, por quien se siente, sino evaluado, al menos "observado".

Restar discursivamente relevancia al dinero ganado por la mujer es también un modo de disminuir el disconfort. Esta estrategia fue observada frecuentemente entre varones y mujeres que han conformado parejas predominantemente homogámicas e hipogámicas. Si los varones otorgaran al ingreso femenino el valor que quizás objetivamente tiene para la familia, estarían reconociendo que "solos no pueden" cumplir con el rol que se espera socialmente que ellos cumplan, lo cual acrecentaría el disconfort.

\section{REFLEXIONES FINALES}

En Argentina, así como en la mayoría de los países de Latinoamérica, el cambio cultural y social propiciado por la Segunda Ola feminista llegó algunos años más tarde que en Europa o Norteamérica, pero se constituyó como un nuevo punto de partida para las mujeres que bogaban por desarrollarse en ámbitos diferentes al estrictamente familiar y ligado a la maternidad. La motivación femenina de abandonar las tareas exclusivas de reproducción no se limitó en nuestro país a cuestiones meramente ideológicas, sino que se sostuvo (y aún se 
sostiene) en una realidad económica que en los años '80 demandó que las mujeres contribuyeran económicamente a los diezmados ingresos familiares (Wainerman, 2007).

Así, nuestra sociedad comenzó a ser testigo del avance de las mujeres en campos antes restringidos a los varones. Sin embargo, los varones no se acercaron significativamente hacia el polo de la reproducción, tendiendo a "resistir" y proteger activamente sus espacios tradicionales basados en la producción, el dominio social (productivo y público), así como la jefatura en el ámbito familiar. La tendencia a "retornar" a los espacios de género tradicionales es observable en las expresiones de los varones entrevistados, aún entre aquellos que se muestran más adaptados a la realidad que proponen los nuevos roles femeninos, y a nuevos modelos de distribución sexual del trabajo.

El avance femenino ubicó además a los varones en una situación de riesgo al tener que asumir tareas para las que no habían sido educados, que les han sido señaladas como de menor jerarquía (por ser femeninas) y que ellos aún perciben como "peligrosas", en términos identitarios (Montesinos, 2002).

Así, el disconfort de género se acrecienta para los varones cuando se sienten impulsados a realizar tareas que entienden como feminizantes, y que atentan contra una imagen de masculinidad que les ha costado gran esfuerzo adquirir y conservar. Aquí surge la dimensión externa del disconfort, ya que muchos de los entrevistados dijeron sentirse observados y atacados por sus congéneres, que los señalan como "poco hombres" por ocuparse de tareas de reproducción que no son consideradas masculinas. Las ocupaciones domésticas entran para ellos en el campo de las representaciones de la femineidad, y aún cuando se esfuerzan por justificar la necesidad de su participación en los trabajos domésticos (por una cuestión de justicia o por ser "políticamente" correctos), los sienten extraños y poco viriles. Las burlas de sus congéneres por adentrarse en el rol de reproducción y hacer "cosas de mujeres", no hacen más que incrementar la sensación de disconfort, ya que ponen en duda la masculinidad.

Una masculinidad ganada con sacrificio y cuidada con esmero supone para los varones un capital simbólico efímero, que corre peligro de desvanecerse en cualquier momento a partir de la falta de empleo, la pérdida del poder al interior de la pareja (al pasar una pareja de ser hipergámica a ser homogámica o hipogámica), la enfermedad o la vejez (Kimmel, 1987).

También en la dimensión externa surge la doble demanda femenina (de la pareja), que requiere que el varón cumpla con el rol de proveedor principal del hogar pero que además contribuya con las actividades de reproducción. Este requerimiento doble que apunta al desempeño del varón en espacios que percibe como contradictorios, le produce incertidumbre en tanto no tiene certezas respecto de cuál es el rol en el que es valorado, y qué se espera de él como sujeto - varón.

La doble demanda de la pareja también surge como disparador del disconfort entre las mujeres, cuando los varones restan valor a las tareas relacionadas con la reproducción, pero a la vez demandan a las mujeres que permanezcan más tiempo en el hogar. Esta contradicción entre la exaltación de la figura de la mujer-madre y la desvalorización del trabajo doméstico, resulta en una incertidumbre que acrecienta la incomodidad femenina.

Los efectos de cambio generados por la Segunda Ola han modificado sustancialmente la vida de las mujeres en la mayoría de los países occidentales, y los discursos relacionados con la igualdad y un equilibrio más justo entre los géneros se imponen como legítimos. Sin embargo, los esquemas normativos referidos a mujeres y varones, que determinan lo que es correcto pensar, hacer y esperar, se encuentran internalizados de modo tal que se resisten a dar paso a las representaciones emergentes en torno a la necesidad de reinterpretar lo que 
significa socialmente ser varón o ser mujer. Esta durabilidad de las representaciones tradicionales da cuenta de lo que Catalina Wainerman ha llamado "revolución estancada" (2007); aún cuando los avances en la organización de los espacios sociales y familiares es notable, ciertas condiciones objetivas en las que se gestan los habitus de los agentes sociales parecen contribuir a ralentizar los cambios en las normas de género.

\section{BIBLIOGRAFÍA}

BOURDIEU, P. (2007): El sentido práctico, Buenos Aires, Siglo XXI.

- (2000): La dominación masculina, Barcelona, Anagrama.

- (1999): Meditaciones pascalianas, Barcelona, Anagrama.

- (1988): La distinción. Criterios y bases sociales del gusto, Madrid, Taurus.

CARBONERO GAMUNDI, M. y S. LEVIN (1998) (comp.): Entre familia y trabajo - Relaciones, conflictos y políticas de género en Europa y América Latina, Rosario, Homo Sapiens.

FRASER, N. (1997): Iustitia interrupta: reflexiones críticas desde la posición postsocialista, Bogotá, Siglo de Hombres.

KIMMEL, M. (1987): Changing men: new directions in research on men and masculinity, London, Sage. MAXWELL, J. (1996): Qualitative research design. An Interactive Approach, Londres, Sage.

MAYOBRE, P. (2004): "La construcción de la identidad personal en una cultura de género", Creatividad feminista, disponible: www.creatividadfeminista.org/articulos/2004/sex04_purificacion .htm. [consulta: 02/05]

MERLINO, A. (2009): Investigación cualitativa en Ciencias sociales: temas, problemas y aplicaciones, Buenos Aires, Cengage Learning.

MONTESINOS, R. (2002): Las rutas de la masculinidad, ensayos sobre el cambio cultural y el mundo moderno, Barcelona, Gedisa.

MOZEJKO, T. y COSTA, R. (comp.) (2002): Lugares del decir - Competencia social y estrategias discursivas, Rosario, Homo Sapiens.

OLABUÉNAGA RUIZ, J. (2003): Metodología de la investigación cualitativa, Bilbao, Deusto.

STRAUSS, A. y J. CORBIN (2002): Bases de la Investigación cualitativa, Medellín, U. Antioquía.

VALLES, M. (2003): Técnicas cualitativas de investigación social. Reflexión metodológica y práctica profesional, Madrid, Síntesis.

VIEYTES, R. (2004): Metodología de la investigación en organizaciones, mercado y sociedad. Epistemología y Técnicas, Buenos Aires, De las Ciencias.

WAINERMAN, C. (2007): "Familia, trabajo y relaciones de género", en Carbonero Gamundi, M. y S. Levin (comp.): Entre familia y trabajo - Relaciones, conflictos y políticas de género en Europa y América Latina, Rosario, Homo Sapiens.

\section{Breve currículo:}

\section{Alejandra Martínez}

Doctora en Ciencias Sociales por la Universidad de Buenos Aires. Realiza estudios de género y familia desde el enfoque de la metodología cualitativa. Recientemente ha publicado: "Masculinity, poverty and violence in Argentina - Analyzing children's discourse" (2009) en Amsterdam Social Science, Ámsterdam, "Distribución y reconocimiento, la doble subalternidad de las mujeres pobres" en Polémicas en torno al modelo teórico de Nancy Fraser, A. Boria y P. Morey (comp.) Buenos Aires, 2010. "La investigación cualitativa en el ámbito de las comunicaciones: un estudio en recepción en niños, a partir de la adaptación de la técnica del grupo de enfoque". En Aldo Merlino (coord.) Investigación cualitativa en ciencias sociales: temas y problemas, Cengage, Buenos Aires, 2009. 